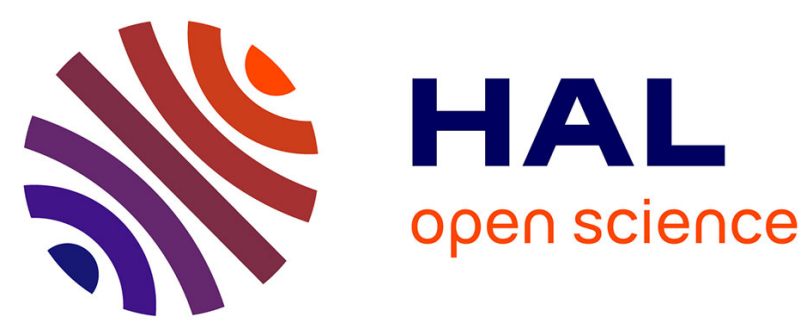

\title{
Bottleneck Prediction Using the Active Period Method in Combination with Buffer Inventories
}

\author{
Christoph Roser, Kai Lorentzen, David Lenze, Jochen Deuse, Ferdinand \\ Klenner, Ralph Richter, Jacqueline Schmitt, Peter Willats
}

\section{- To cite this version:}

Christoph Roser, Kai Lorentzen, David Lenze, Jochen Deuse, Ferdinand Klenner, et al.. Bottleneck Prediction Using the Active Period Method in Combination with Buffer Inventories. IFIP International Conference on Advances in Production Management Systems (APMS), Sep 2017, Hamburg, Germany. pp.374-381, 10.1007/978-3-319-66926-7_43 . hal-01707303

\section{HAL Id: hal-01707303 https://hal.inria.fr/hal-01707303}

Submitted on 12 Feb 2018

HAL is a multi-disciplinary open access archive for the deposit and dissemination of scientific research documents, whether they are published or not. The documents may come from teaching and research institutions in France or abroad, or from public or private research centers.
L'archive ouverte pluridisciplinaire HAL, est destinée au dépôt et à la diffusion de documents scientifiques de niveau recherche, publiés ou non, émanant des établissements d'enseignement et de recherche français ou étrangers, des laboratoires publics ou privés. 


\title{
Bottleneck Prediction Using the Active Period Method in Combination with Buffer Inventories
}

\author{
Christoph Roser ${ }^{1}$, Kai Lorentzen ${ }^{2}$, David Lenze ${ }^{3}$ Jochen Deuse $^{3}$, Ferdinand Klenner ${ }^{4}$, \\ Ralph Richter ${ }^{5}$, Jacqueline Schmitt ${ }^{3}$, Peter Willats ${ }^{3}$ \\ ${ }^{1}$ Karlsruhe University of Applied Sciences, Karlsruhe, Germany \\ (Corresponding Author) \\ christoph.roser@hochschule-karlsruhe.de \\ ${ }^{2}$ Robert Bosch GmbH, Stuttgart, Germany \\ kai.lorent zen@de.bosch.com \\ ${ }^{3} \mathrm{TU}$ Dortmund University, Dortmund, Germany \\ sekretariateips.tu-dortmund. de \\ ${ }^{4}$ BMW Group, Munich, Germany \\ ferdinand. klenner@bmw . de \\ ${ }^{5}$ Robert Bosch GmbH, Stuttgart, Germany \\ ralph.richter@de.bosch.com
}

\begin{abstract}
Knowing the bottleneck is one of the keys to improving a production system. The active period method is one approach to detect shifting bottlenecks that most other bottleneck detection methods have problems with. Yet, like many other methods, these detections are limited to detecting the past and present bottlenecks. In this paper, we combined the active period method with the buffer inventories and free buffer spaces of the adjacent inventories to statistically predict not only an upcoming change of the bottleneck, but also where the bottleneck will move to.
\end{abstract}

Keywords: bottleneck detection, inventory, bottleneck prediction, active period method, bottleneck walk

\section{Introduction and Literature Review}

Bottleneck ${ }^{1}$ detection is the key to improving output in any production system, and also the basis to predict the shifting of a bottleneck. Only the improvement of the throughput of a bottleneck process will lead to an improvement of the throughput of the entire system. Unfortunately, real-world systems are dynamic and rarely have a single, permanent bottleneck. Instead, the bottleneck shifts between different processes. Therefore, we define the bottleneck as follows:

${ }^{1}$ Please note that throughout this paper we will occasionally abbreviate "bottleneck" as "BN" in graphics. 
Bottlenecks are processes that influence the throughput of the entire system. The larger the influence, the more significant the bottleneck [1] [2] [3] [4].

To detect shifting bottlenecks, it is necessary to determine how the momentary bottleneck changes over time. Hence, it is necessary to determine the momentary or real-time bottleneck. Due to the nature of the shifting bottleneck, however, it is not possible to determine momentary bottlenecks using long-term averages.

Unfortunately, many bottleneck detection methods presented in academic publications or used in industry are based on long-term averages, as for example the average cycle time or utilization [4] [5] [6] [7] [8], total waiting time [5], average waiting time [9], average length of the queue [10] [11], or combinations thereof [12], and average time blocked or starved [13] [14] [15]. Overviews of different bottleneck detection methods can be found, for example, in [3] [16] [17].

There are very few methods available that are actually able to detect the momentary bottlenecks. These are the active period method and the bottleneck walk. The active period method determines the momentary bottleneck through the process that has currently the longest period without interruption through waiting times (the active period) [18] [19] and a simplified variant thereof [20]. This method is very precise but has high requirements on the quality of the data.

The bottleneck walk looks at both processes and inventories to determine the direction in which the bottleneck is likely to be found [1] [21]. This approach is very well suited for practical use in flow lines, although it has difficulties with job shop-type production systems. These two methods consistently outperform other bottleneck detection methods [16] [3] [22]. Within this paper we will use the active period method, and hence will present this approach in more detail below.

\section{Need for and Use of Predictive Methods}

Detecting the past and current bottleneck is one step to improving system output. Yet another major step is to predict the behavior of the bottleneck in the future. If a shift in the bottleneck can be predicted before it happens, then it is possible to counteract this change and prevent a shift. While this will not eliminate the bottleneck in the system, it can avoid or reduce the negative influence of shifting bottlenecks among each other.

Wedel et al investigated the effect of the buffer preceding the bottleneck on the prediction of the change in the bottleneck [23] [24]. However, while they investigate both upstream and downstream buffers, they do not investigate where the bottleneck shifts to.

\section{The Active Period Method}

As this bottleneck prediction method is based on the active period method, we will briefly describe the bottleneck walk. More details on this method can be found in [18] [19], and an evaluation of its abilities in [16] [3] [22] [25]. 
The active period method is based on the status of the processes across time. For the duration of the observation, the status of all processes is monitored. While the process can be in many possible status situations, like working, repair, waiting for parts, changeover, maintenance, waiting for transport, etc., these are aggregated into two groups: 1) Waiting on another process, called inactive; and 2) NOT waiting on another process, called active. These active periods are plotted as shown in Fig. 1 for a simple example using four processes in sequence.

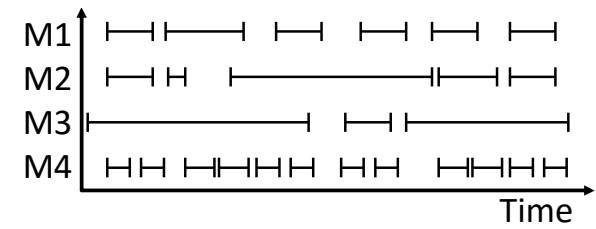

Fig. 1. Simple example of the active periods for two processes

At any given time, the process with the longest active period is the bottleneck. The bottleneck shifts when one longest period overlaps with the next longest active period. Fig. 2 shows how the bottleneck changes from machine M3 to M2 and back for the simple example from Fig. 1.

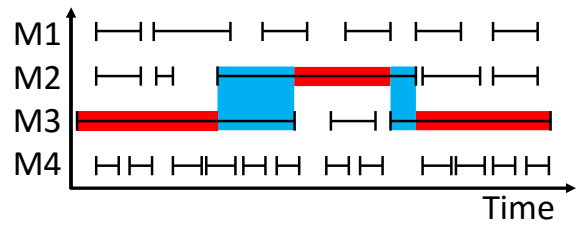

Fig. 2. Simple example of the active periods for two processes showing the bottleneck shift

This active period method is very precise and well suited for many different kinds of production systems. Its drawback is the data requirement, as a continuous data stream from all processes is necessary.

\section{$4 \quad$ Adapting the Active Period Method for Prediction}

The active period method analyses an entire data set from beginning to end. Hence, it must be adapted before it can be used for bottleneck prediction.

\subsection{Floating Observation}

The major difference is that for a bottleneck prediction, we do not know the future behavior of the production system (otherwise no prediction would be needed in the first place). Fig. 3 shows the example from Fig. 1, although with an unknown future development. 


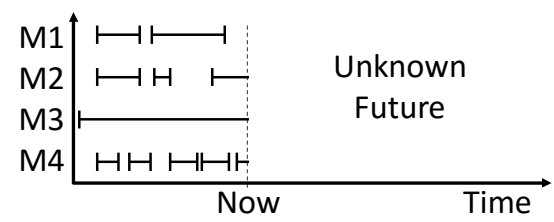

Fig. 3. Simple example with active periods up to the current moment of observation

Here, too, the current bottleneck is the process with the longest active period until now. Unfortunately, we do not know a shift until it happens. However, we do know which process would be the runner-up. Fig. 4 shows the current bottleneck M3 in red and the possible next bottleneck M2 in blue. If the process marked in blue will become the bottleneck or not is not visible from this data until it happens.

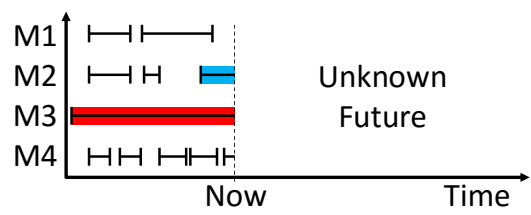

Fig. 4. Floating bottleneck observation and runner-up

\subsection{Prediction of Bottleneck Shift Event}

Hence, the second problem is to predict when the bottleneck will shift. A bottleneck will shift if the so-far-longest active period is interrupted. This can happen in two cases: the process with the longest active period so far runs out of parts or runs out of space to store parts. These two situations are commonly known as starved and blocked.

The likelihood of this happening can be directly observed from the buffer before and after the currently longest active process. If the buffer before the currently longest active process starts to run empty, then the risk of interruption through starving increases. If the buffer after the currently longest active process starts to run full, then the risk of interruption through blocking increases.

\subsection{Prediction of Future Bottleneck}

When a bottleneck shifts, the future bottleneck is the process that at the moment before the shift has the second-longest active period, and is therefore the longest active period after the shift. The example in Fig. 4 shows the possible future bottleneck as process M2.

\section{$5 \quad$ Simulation Verification}

The above approaches have been verified using simulation data. Our system consisted of eight processes in sequence, as shown in Fig. 5, always separated by a 
buffer with a capacity of 10 . There is infinite demand and supply at the system boundaries. The cycle time of the processes are exponentially distributed with a mean of 2 , except for process P3 and P6, which have a mean of 3. Hence, processes P3 and P6 are likely to be the bottlenecks. The simulation was run for 8000 time units.

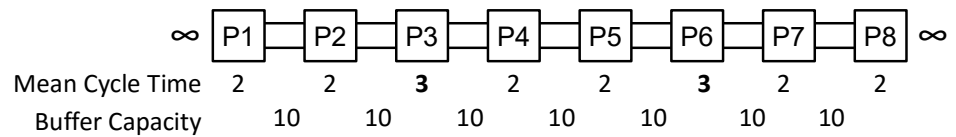

Fig. 5. Simulation example

Fig. 6 shows the results of the active period analysis. As expected, the bottleneck changes mainly between processes P3 and P6. Overall, P3 was the sole bottleneck $29 \%$ of the time and shifting for $31 \%$, whereas P6 was the sole bottleneck $39 \%$ of the time and shifting for $30 \%$. All other processes have a negligible impact on the system performance with below $2 \%$.

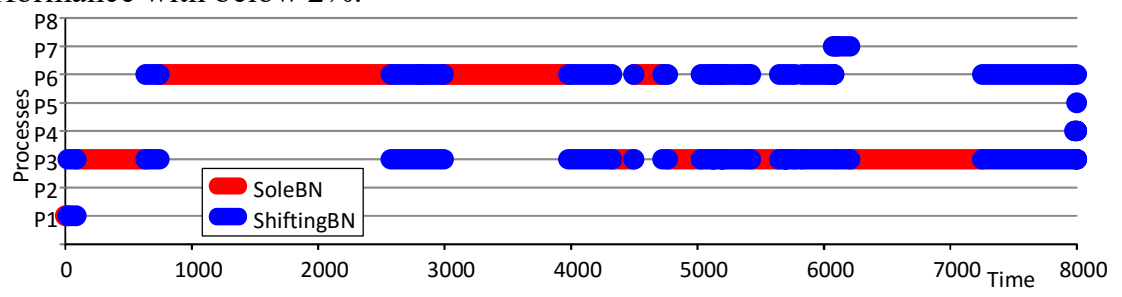

Fig. 6. Active periods of simulation example

For all bottleneck shifts within the simulation, we plotted the behavior of the buffer before and after the currently longest active period up to the moment of the actual shift. This is shown in Fig. 7. The x-axis is the negative time until the shift of the bottleneck away from the currently longest active period. The 22 graphs overlap each other partially. All of these graphs start when the bottleneck shifted to the process, and end when the bottleneck shifts away (time 0 on the $\mathrm{x}$-axis). The y-axis shows the available parts in the buffer in front of the bottleneck on the top half, and the available free spaces inverted on the bottom half of the graph.

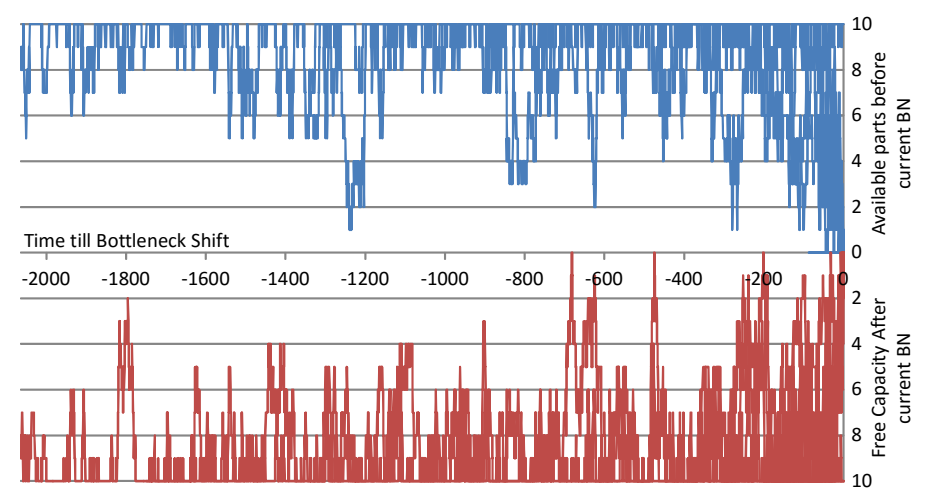

Fig. 7. Critical buffers before and after longest active period before switch 
The results are very concise. When the bottleneck shifts to the process observed, the process was already active long enough to be the second-longest active period until the moments before the shift. Hence, the buffers before the process have a tendency to be full and the buffer after the process have a tendency to be empty.

Interestingly enough, this changes only when a bottleneck shift approaches. With very few exceptions, a nearly empty buffer before or a nearly full buffer after the process quickly leads to a shift in the bottleneck.

This is also confirmed through another analysis. Fig. 8 shows the mean time until the shift of the bottleneck depending on the available parts before the bottleneck and the available spaces after the bottleneck. It is clearly visible that as the number of available parts or spaces approaches zero, the mean time until the next bottleneck shift is significantly reduced. Fig. 8 also shows the time that is covered in a worst case, which is directly related to the number of parts or number of free spaces in the adjacent buffer. This worst case is only a fraction of the mean time to shift, as there are usually new parts constantly arriving and leaving the buffer before and after the current bottleneck respectively.

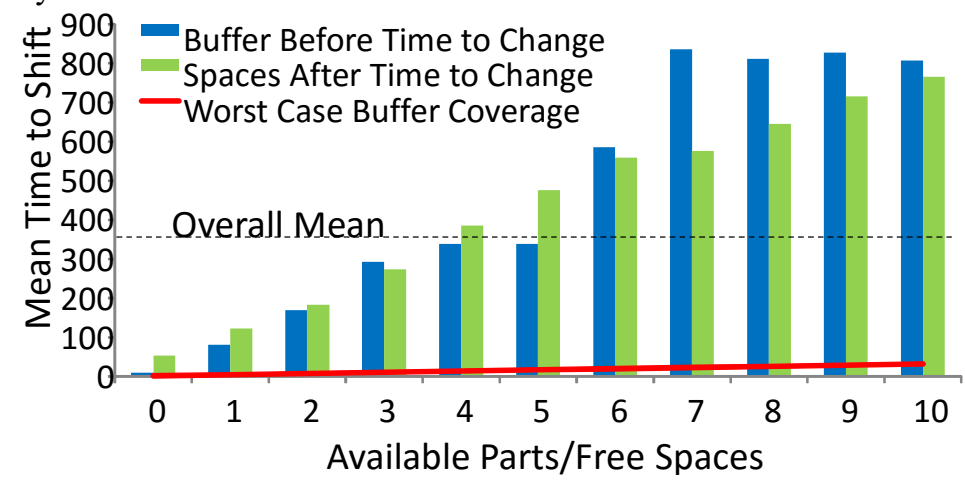

Fig. 8. Mean time until shift for different inventory levels

\section{Summary}

Overall, the active period method can easily be adapted to detect the current bottleneck in real time. Furthermore, by simply analyzing the buffers before and after the current bottleneck, it is possible to estimate if a shift may occur sooner or later. Finally, the process to which the bottleneck will shift to is also already known as the process with the second-longest active period before the shift. Hence, the presented method allows not only the detection but also the prediction of bottleneck shifts in real time. Similar to the normal active period method, the approach is very accurate and intuitive, but requires detailed process data of all processes in the system. 


\section{$7 \quad$ References}

[1] C. Roser, K. Lorentzen, and J. Deuse, "Reliable Shop Floor Bottleneck Detection for Flow Lines Through Process and Inventory Observations: The Bottleneck Walk," Logist. Res., vol. 8, no. 7, Oct. 2015.

[2] C. Romeser and C. Roser, "Direction of the Bottleneck in Dependence on Inventory Levels," in Proceedings of the International Conference on the Advances in Production Management System, Iguassu Falls, Brazil, 2016.

[3] C. Roser and M. Nakano, "A Quantitative Comparison of Bottleneck Detection Methods in Manufacturing Systems with Particular Consideration for Shifting Bottlenecks," in Proceedings of the International Conference on the Advances in Production Management System, Tokyo, Japan, 2015.

[4] S.-Y. Chiang, C.-T. Kuo, and S. M. Meerkov, "Bottlenecks in Markovian Production Lines: A Systems Approach," IEEE Trans. Robot. Autom., vol. 14, no. 2, pp. 352-359, 1998.

[5] A. M. Law and D. W. Kelton, Simulation Modeling \& Analysis, 2nd ed. McGraw Hill, 1991.

[6] "Knessl and Tier - 1998 - Asymptotic approximations and bottleneck analysis .pdf." .

[7] P. J. Schweitzer, G. Serazzi, and M. Broglia, "A survey of bottleneck analysis in closed networks of queues," in Performance Evaluation of Computer and Communication Systems, L. Donatiello and R. Nelson, Eds. Springer Berlin Heidelberg, 1993, pp. 491-508.

[8] S.-Y. Chiang, C.-T. Kuo, and S. M. Meerkov, "c-Bottlenecks in Serial Production Lines: Identification and Application," Math. Probl. Eng., 2002.

[9] P. K. Pollett, "Modelling congestion in closed queueing networks," Int. Trans. Oper. Res., vol. 7, no. 4-5, pp. 319-330, Sep. 2000.

[10] S. R. Lawrence and A. H. Buss, "Shifting Production Bottlenecks: Causes, Cures, and Conundrums," J. Prod. Oper. Manag., vol. 3, no. 1, pp. 21-37, 1994.

[11] L. Li, Q. Chang, J. Ni, G. Xiao, and S. Biller, "Bottleneck Detection of Manufacturing Systems Using Data Driven Method," in IEEE International Symposium on Assembly and Manufacturing, 2007. ISAM '07, 2007, pp. 76-81.

[12] G. F. Elmasry and C. J. McCann, "Bottleneck discovery in large-scale networks based on the expected value of per-hop delay," in 2003 IEEE Military Communications Conference, 2003. MILCOM '03, 2003, vol. 1, p. 405-410 Vol.1.

[13] C.-T. Kuo, J.-T. Lim, and S. M. Meerkov, "Bottlenecks in Serial Production Lines: A System-Theoretic Approach," Math. Probl. Eng., vol. 2, pp. 233-276, 1996.

[14] L. Li, Q. Chang, and J. Ni, "Data driven bottleneck detection of manufacturing systems,” Int. J. Prod. Res., vol. 47, no. 18, pp. 5019-5036, Jun. 2009. 
[15] S. Sengupta, K. Das, and R. P. VanTil, "A New Method for Bottleneck Detection," in Proceedings of the 40th Conference on Winter Simulation, Miami, Florida, 2008, pp. 1741-1745.

[16] E. Lima, L. Chwif, and M. R. P. Barreto, "Metodology for selecting the best suitable bottleneck detection method," in Simulation Conference, 2008. WSC 2008. Winter, 2008, pp. 1746-1751.

[17] Y. Wang, Q. Zhao, and D. Zheng, “-," J. Syst. Sci. Syst. Eng., vol. 14, no. 3, pp. 347-363, Sep. 2005.

[18] C. Roser, M. Nakano, and M. Tanaka, "Detecting Shifting Bottlenecks," in International Symposium on Scheduling, Hamamatsu, Japan, 2002, pp. 59-62.

[19] C. Roser, M. Nakano, and M. Tanaka, "Shifting Bottleneck Detection," in Winter Simulation Conference, San Diego, CA, USA, 2002, pp. 1079-1086.

[20] C. Roser, M. Nakano, and M. Tanaka, "A Practical Bottleneck Detection Method," in Proceedings of the Winter Simulation Conference, Arlington, Virginia, USA, 2001, vol. 2, pp. 949-953.

[21] C. Roser, K. Lorentzen, and J. Deuse, "Reliable Shop Floor Bottleneck Detection for Flow Lines through Process and Inventory Observations," in Proceedings of the Robust Manufacturing Conference, Bremen, Germany, 2014.

[22] J. Deuse, D. Lenze, F. Klenner, and T. Friedrich, "Manufacturing Data Analytics zur Identifikation dynamischer Engpässe in Produktionssystemen mit hoher wertschöpfender Variabilität," in Megatrend Digitalisierung - Potenziale der Arbeits- und Betriebsorganisation, Gito, 2016.

[23] M. Wedel, M. von Hacht, R. Hieber, J. Metternich, and E. Abele, "Real-time Bottleneck Detection and Prediction to Prioritize Fault Repair in Interlinked Production Lines," Procedia CIRP, vol. 37, pp. 140-145, Jan. 2015.

[24] M. Wedel, P. Noessler, and J. Metternich, "Development of bottleneck detection methods allowing for an effective fault repair prioritization in machining lines of the automobile industry," Prod. Eng., vol. 10, no. 3, pp. 329-336, Jun. 2016.

[25] C. Roser, M. Nakano, and M. Tanaka, "Comparison of Bottleneck Detection Methods for AGV Systems," in Winter Simulation Conference, New Orleans, Louisiana, USA, 2003, pp. 1192-1198. 